\title{
QUANTUM FIELD THEORY IN NINETY MINUTES
}

\author{
PAUL FEDERBUSH
}

These notes grew out of a ninety-minute lecture delivered in a seminar at the University of Michigan. The audience consisted of mathematicians with a very wide spectrum of research interests. In fact, there was only one other mathematical physicist present. We here attempt to preserve some of the casual flavor of the live seminar. There will be some generalizations, imprecise statements, and disputable implications. These are natural in trying to cover so broad an area as Euclidean quantum field theory, so briefly, demanding no specialized background. On the other hand, we proudly hold up to the experts the accomplishment of here presenting a complete, precise, rigorous definition of the two-dimensional quantum field theory $p(\phi)_{2}$, easily accessible to most graduate students in mathematics. The concepts of cutoffs, renormalization, and perturbation series are touched on, as are some of the features of more complicated theories. Recent theoretical developments have made possible the simplicity and elegance of the present treatment.

Defining a Euclidean quantum field theory (as pioneered by E. Nelson) is exactly the problem of making sense of an initially only formally defined functional integral. We start by listing several example theories in (space-time) dimensions one through four.

I. A particle moving in the potential $V(x)$ (a one-dimensional field theory). Here one integrates over the space whose points are paths,

$$
\phi(x): R^{1} \rightarrow R^{1} .
$$

One should here be impressed with the problem of establishing an integral, or measure, on such a huge, infinite-dimensional space. We put a weighting on the path, $\phi(x)$, given by $e^{-S(\phi)}$,

$$
S(\phi)=\int\left[\frac{1}{2}\left(\frac{d \phi}{d x}\right)^{2}+V(\phi)\right] d x .
$$

Received by the editors December 3, 1986.

1980 Mathematics Subject Classification (1985 Revision). Primary 81E10; Secondary 81C20.

This work was supported in part by the National Science Foundation under Grant No. PHY-85-02074. 
$S(\phi)$ is called the action. We desire to make sense of formal integrals such as

$$
\int \mathscr{D}_{\phi} e^{-S(\phi)} \not(\phi)
$$

where, formally,

$$
\int \mathscr{D}_{\phi}=\prod_{x}\left(\int_{-\infty}^{\infty} d \phi(x)\right)
$$

integrates over all possible functions (paths). We will assume $V$ is continuous and satisfies

$$
V \geqslant c
$$

for some $c$.

II. The two-dimensional $p(\phi)_{2}$ field theory. The integral is now over an even larger space of functions; a point in the space is a mapping

$$
\phi(x): R^{2} \rightarrow R \text {. }
$$

The (formal) action is

$$
S(\phi)=\int\left[\frac{1}{2}(\vec{\nabla} \phi)^{2}+\frac{M^{2}}{2} \phi^{2}+\lambda p(\phi)\right] d^{2} x
$$

where $M$ is the mass, assumed nonzero, and $p(\phi)$ is a polynomial that is bounded below,

$$
p(\phi) \geqslant c .
$$

III. The three-dimensional $\phi_{3}^{4}$ field theory. We have

$$
\phi(x): R^{3} \rightarrow R
$$

and a (formal) action

$$
S(\phi)=\int\left[\frac{1}{2}(\vec{\nabla} \phi)^{2}+\frac{M^{2}}{2} \phi^{2}+\lambda\left(a \phi^{4}+b \phi^{3}+c \phi\right)\right] d^{3} x
$$

with $a>0$.

IV. The four-dimensional Yang-Mills theory, $Y-M_{4}$. Here we consider connections (or potentials) $A_{\mu}(x)$, mappings

$$
A_{\mu}(x): R^{4} \rightarrow \mathscr{G}
$$

into the Lie algebra of a simple compact Lie group. The action is taken as

$$
S\left(A_{\mu}\right)=\int\left|F_{\mu v}(x)\right|^{2} d^{4} x
$$

with $F_{\mu v}$ the curvature (or field) due to $A_{\mu}(x)$. For those to whom the algebra of this example is foreign, we emphasize that in these notes we only tangentially discuss this example.

We first note that the dimension of each field theory is the dimension of the domain space of the corresponding $\phi(x)$ (or $A_{\mu}(x)$ ), not of the range. In fact the examples I-III may be easily modified to have the dimension of the range 
greater than one, without substantially increasing the difficulty of their construction.

We by no means claim these are the only interesting examples, but they are very representative. We mean them to indicate the increasing difficulty of constructing theories with increasing dimension. Thus one does not know how to modify II to replace the polynomial $p$ with a merely continuous function such as $V$ of I. And there are strong arguments that the fourth-order polynomial in III may not be replaced by a higher-order polynomial, as may occur in II. (Again one does not know how then to define the functional integral in a satisfactory way. We do not here detail the set of requirements on the definition - the axioms of Euclidean field theory.) Finally it is believed that theories such as in III do not exist in four dimensions, that the Yang-Mills theory is the simplest four-dimensional theory.

As a brief aside we note that the measure constructed for I will live on the continuous functions, but in the other cases the measure will be supported on some space of distributions. This indicates why such expressions as

$$
\int \phi^{4}(x) d^{2} x
$$

may be difficult to deal with, involving the pointwise multiplication of distributions. One could imagine an expression such as

$$
\int d^{2} x_{1} \cdots d^{2} x_{4} f\left(x_{1}, x_{2}, x_{3}, x_{4}\right) \phi\left(x_{1}\right) \cdots \phi\left(x_{4}\right)
$$

replacing (13), with $f$ smooth. Such nonlocal modifications have been studied by physicists, but local expressions such as (13) are the ones that lead to satisfactory field theories. The "renormalizations" we will see below are necessitated by the difficulty of defining expressions such as (13). Our route will not involve looking at any questions of measure.

The functional integral of I was defined long ago as the well-known Wiener integral. Examples II and III were first fully treated primarily by the efforts of Glimm and Jaffe (who established the discipline of constructive field theory). The Yang-Mills theory $Y-M_{4}$ is still undefined, but we may expect substantial progress here within a few years.

We steer our discussion to the $p(\phi)_{2}$ theory. Our formalism immediately provides an alternate derivation of the Wiener measure of $I$, which we do not pursue; we later make some remarks on the corresponding treatment of III.

In II and III one singles out the free action

$$
S_{0}=\int d x\left[\frac{1}{2}(\vec{\nabla} \phi)^{2}+\frac{M^{2}}{2} \phi^{2}\right]
$$

and in some sense treats the rest of $S$ as a "perturbation." $S_{0}$ is quadratic in $\phi$, and it is necessary now to study such quadratic weightings - to study Gaussian integrals.

In an $N$-dimensional vector space we let $D$ be a strictly positive symmetric matrix. We consider the integral

$$
I(F)=\int d \vec{\alpha} e^{-(1 / 2) \alpha D \alpha} F(\alpha), \quad \text { with } \int d \vec{\alpha}=\prod_{i=1}^{N} \int_{-\infty}^{\infty} d \alpha_{i},
$$


and

$$
\alpha D \alpha=\sum_{i, j} \alpha_{i} D_{i j} \alpha_{j}
$$

$F$ is a suitable function of the $\alpha$ 's. We note a few examples:

$$
I\left(\alpha_{i}\right)=0
$$

by the symmetry of the integrand under $\alpha \rightarrow-\alpha$;

$$
I\left(\alpha_{i} \alpha_{j}\right)=C_{i j} I(1)
$$

where

$$
C=D^{-1}
$$

and is called the covariance. There is no difficulty checking (19); it is actually an immediate consequence of the following more general result.

We assume that $F$ and its first partials are polynomially bounded (actually only that they do not grow as fast as $e^{c|\alpha|^{2}}$ ). We then have

$$
I\left(\alpha_{i} F\right)=\sum_{j} C_{i j} I\left(\frac{\partial F}{\partial \alpha_{j}}\right) .
$$

We refer to (21) as integration by parts. We note that in addition to (19), I of any polynomial may be evaluated by inductive use of (21). (We use (21) with $F=\alpha_{j}$ to derive (19).) (21) follows easily from the identity

$$
\alpha_{i} e^{-(1 / 2) \alpha D \alpha}=-\sum_{j} C_{i j} \frac{\partial}{\partial \alpha_{j}}\left(e^{-(1 / 2) \alpha D \alpha}\right)
$$

and (usual) integration by parts.

Given a linear function of the $\alpha$ 's

$$
l=\sum a_{i} \alpha_{i}
$$

we now define the Wick ordering or normal ordering of powers of $l$ (indicated by dots)

$$
: l^{s}:=l^{s}+\sum_{j=1}^{s} b_{j} l^{s-j}
$$

where the $b_{j}$ are determined by (a Gram-Schmidt procedure type requirement)

$$
I\left(: l^{s}: l^{r}\right)=0 \text { if } r<s .
$$

We note that the $b_{j}=0$ for odd $j$. As an example, we consider

$$
: l^{2}:=l^{2}-c_{l} \text {, }
$$

where

$$
0=I\left(: l^{2}: l^{0}\right)=\left(l^{2}-c_{l}\right)=I(1)\left(\sum_{i, j} a_{i} C_{i j} a_{j}-c_{l}\right)
$$

shows

$$
c_{l}=a C a
$$


$: l^{s}:$ is a renormalization of $l^{s}$. (As a final note on the construction of the $: l^{s}$, , we point out that the polynomials in (24) are Hermite polynomials.)

In the Appendix we describe a countable discrete set of functions $\left\{u_{k}(x)\right\}$ that are in a suitable sense complete. In fact the $u(x)$ are of the form

$$
u_{k}(x)=\left(\frac{1}{\sqrt{-\Delta+M^{2}}} \psi_{k}^{\prime}\right)(x),
$$

where the $\left\{\psi_{k}^{\prime}\right\}$ form an o.n. basis of $L^{2}\left(R^{d}\right)$ ( $d$ is the dimension of the theory we are working with, 2 for $\left.p(\phi)_{2}\right)$. $\left(-\Delta+M^{2}\right)^{-1 / 2}$ is an integral operator defined from the Laplacian. The reader unfamiliar with such operators is here advised to simply take the $u_{k}(x)$ as a given set of functions, suitably complete. The choice of the basis $\left\{\psi_{k}^{\prime}\right\}$ and thus of the $\left\{u_{k}\right\}$ is not arbitrary; the theorem we quote below depends on certain detailed properties of the basis. (The renormalization group of $\mathrm{K}$. Wilson is implicitly incorporated in our work by its natural action on this basis. Exploiting this naturally leads into the fundamental work of Gawedzki and Kupiainen.) We expand

$$
\phi(x)=\sum \alpha_{k} u_{k}(x)
$$

and choose to describe the field (function) $\phi(x)$ in terms of the variables $\left\{\alpha_{k}\right\}$. Our functional integral becomes

$$
\int \mathscr{D}_{\phi}=\prod_{k} \int_{-\infty}^{\infty} d \alpha_{k}
$$

where we have traded one formal expression for another. But the discrete product on the right side of (31) is "more reasonable" than the corresponding product in (4). We may view the change from (4) to (31) as a change in variables. The corresponding (formal) Jacobian is a constant (it is a linear change of variables) and is neglected.

We now let $A$ be any finite subset of the $\left\{\alpha_{k}\right\}$. We define the "cutoff field" $\phi_{A}(x)$

$$
\phi_{A}(x)=\sum_{k \in A} \alpha_{k} u_{k}(x) .
$$

We note that (referring to (15))

$$
S_{0}^{A} \equiv S_{0}\left(\phi_{A}(x)\right)=\sum_{k_{1}, k_{2} \in A} \alpha_{k_{1}} D_{k_{1}, k_{2}}^{A} \alpha_{k_{2}}=\alpha D^{A} \alpha,
$$

where $D^{A}$ is a strictly positive symmetric matrix on a space of dimension the number of elements in $A$. (We suppress the fact that $D^{A}$ is the identity matrix.) We let $: l^{s}:{ }_{A}$ denote the Wick ordering of $l^{s}, l$ linear in elements in $A$, with respect to the Gaussian integral defined by $D^{A}$. We let

$$
S=S_{0}+S_{\mathrm{I}}
$$

with (from (7))

$$
S_{\mathrm{I}}=\lambda \int p(\phi) d^{2} x=\lambda \sum_{i=0}^{N} c_{i} \int \phi^{i}(x) d^{2} x
$$


(The subscript I stands for interaction.) We now seek to define $S^{A}$, the action for functions restricted to the subspace generated by $\left\{u_{k}(x)\right\}_{k \in A}$. We already have defined $S_{0}^{A}$; we seek $S_{\mathrm{I}}^{A}$ so that

$$
S^{A}=S_{0}^{A}+S_{\mathrm{I}}^{A} .
$$

$S_{\mathrm{I}}^{A}$ is obtained from $S_{\mathrm{I}}$ in two steps. The first step changes $S_{\mathrm{I}}$ in (35) to

$$
\lambda \sum_{i=0}^{N} c_{i} \int \phi_{A}^{i}(x) d^{2} x .
$$

We have performed cutoffs in going from (35) to (37). In the next step we change (37) to

$$
S_{\mathrm{I}}^{A} \equiv \lambda \sum_{i=0}^{N} c_{i} \int: \phi_{A}^{i}(x):{ }_{A} d^{2} x .
$$

The step from (37) to (38) is the renormalization of the action. (It is important that terms added in renormalization be formally local.) If we look at the single term : $\phi_{A}^{2}(x):_{A}$ and take the formal limit as $A$ becomes the infinite set of all $u_{k}$ we write (from (26)-(28))

$$
\begin{aligned}
: \phi_{A}^{2}(x):_{A} & \rightarrow \phi^{2}(x)-\lim _{A \rightarrow \Omega} \sum_{k_{1}, k_{2} \in A} u_{k_{1}}(x) C_{k_{1}, k_{2}}^{A} u_{k_{2}}(x) \\
& \rightarrow \phi^{2}(x)-\infty
\end{aligned}
$$

The value of the limit is easily established-with a little technique. This is an infinite renormalization - but note that in our procedure we see no infinities.

We assume our "observables," the $\not(\phi)$ of (3), are polynomials in the $\alpha_{k}$. We define

$$
[\not]_{A}=\prod_{k \in A}\left(\int_{-\infty}^{\infty} d \alpha_{k}\right) e^{-S^{A}} \not h
$$

and

$$
\langle\not\rangle_{A}=[\not h]_{A} /[1]_{A} \text {. }
$$

$[1]_{A}$ is the "normalization", or "partition function". We now state our main theorem for $p(\phi)_{2}$, a prototype for other theories.

MAIN TheOREM. If $\lambda>0$ is sufficiently small, then the following limit exists, for all $p$ as above:

$$
\langle\not p\rangle=\lim _{A>\Omega}\langle\not h\rangle_{A} .
$$

The limit is taken over ascending sequences of sets that eventually exhaust all elements. The same limit is obtained for all such ascending sequences. $\left(\langle\not h\rangle^{A}\right.$ is a net on the directed set of finite subsets of the $u_{k}$. This net converges.)

This theorem establishes expectation values of observables, from which the functional integral and the corresponding measure may be recovered. At this point we claim we have defined the $p(\phi)_{2}$ theory. The main theorem is proven in the formalism of the phase cell cluster expansion. The proof is technical, 
involving the machinery of constructive quantum field theory. We hope some of you are inspired to read the proof. (Our references are given at the end.) The formalism is powerful, and not specialized to the two-dimensional situation.

The main theorem for $\phi_{3}^{4}$ will be identical, but the corresponding $S^{A}$ will be defined in a more complex way. An additional renormalization will be necessary, adding a further term to the $S^{A}$ of the general form

$$
\int d^{3} x \rho^{A}(x) \phi_{A}^{2}(x) .
$$

This is a mass renormalization.

The limit in (43) is very elegant; to one in the field, compellingly so. It treats, on one footing, the infinite volume limit and the removal of the ultraviolet cutoff.

We turn to our final topic, perturbation series, that we treat very cursorily. In physics one is mainly interested in correlation functions such as $\langle\phi(x) \phi(y)\rangle$. This may be defined as

$$
\langle\phi(x) \phi(y)\rangle=\lim _{A \rightarrow \Omega}\left\langle\phi_{A}(x) \phi_{A}(y)\right\rangle_{A} .
$$

(One of the axioms of Euclidean field theory is that the value of such correlation functions as $\langle\phi(x) \phi(y)\rangle$ is invariant if the arguments are changed by a Euclidean translation or rotation.) The perturbation series for this is the series

$$
\langle\phi(x) \phi(y)\rangle=\sum_{0}^{\infty} \lambda^{n} C_{n}(x, y) .
$$

One expects such series to never converge, but to be asymptotic (as has been proved in many cases). In QED, the physical theory of electrons and photons, one believes the corresponding functional integrals do not exist. (QED is not a mathematically consistent field theory.) Yet the formal perturbation series is amazing. Agreements between theory and experiment (between predictions of the first few terms in perturbation series and experiment) have been obtained to one part in $10^{12}$ ! Thus there is some basis for believing in perturbation series predictions-even when the theory is in mathematical purgatory. Because of the limits involved (as we will see below) the definition of the individual terms, $C_{n}(x, y)$ say in (46), is highly nontrivial. These arise evaluated as "Feynman integrals" associated to "Feynman diagrams."

One may "cleanly" expand

$$
\left\langle\phi_{A}(x) \phi_{A}(y)\right\rangle_{A}=\sum_{0}^{\infty} \lambda^{n} C_{n}^{A}(x, y)
$$

(still an asymptotic series), and find

$$
C_{n}(x, y)=\lim _{A>\Omega} C_{n}^{A}(x, y) .
$$

The definition of $C_{n}(x, y)$ as the limit in (48) is very close (identical in spirit) to definitions currently being pursued by such researchers as Gallavotti and Nicolò. But the definition in (48), or the similar expressions in the work of the above-named authors, are not as effective for computation as the limiting 
procedures used by physicists. Though one may verify that different limiting procedures give the same answer, some may be used to do calculations and others are only of theoretical interest. (One has not established all the equivalences one would like; this is a difficult occupation.) In the evaluations of the $C_{n}(x, y)$ via one or another limiting procedure, use of the limiting procedures is called renormalization (a related but different use of the word from one we made before).

We apply the integration by parts identity (21) to get the identity

$$
\left\langle\phi_{A}(x) F\right\rangle_{A}=\sum_{k, k^{\prime} \in A} u_{k}(x) C_{k, k^{\prime}}^{A}\left\langle\left(\frac{\partial F}{\partial \alpha_{k^{\prime}}}-F \frac{\partial S_{\mathrm{I}}^{A}}{\partial \alpha_{k^{\prime}}}\right)\right\rangle_{A} .
$$

We now take the formal limit of (49) term by term.

$$
\text { " }\left\langle\phi_{A}(x) F\right\rangle=\int d z C_{0}(x, z)\left\langle\left(\frac{\delta F}{\delta \phi(z)}-F \frac{\delta S_{\mathrm{I}}^{\Omega}}{\delta \phi(z)}\right)\right\rangle . "
$$

The quotes indicate our embarassment at writing the expression down. This expression has difficulties from $C_{0}(x, z)$ when $x \sim z\left(C_{0}(x, z)\right.$ is as in (46), is called the free propagator, and is singular at $x=z$ ), from the singular formal limit

$$
\text { “ } S_{\mathrm{I}}^{\Omega}=\lim _{A>\Omega} S_{\mathrm{I}}^{A} "
$$

and from the product " $F \delta S_{\mathrm{I}}^{\Omega} / \delta \phi(z)$ ". We have used the formal "functional derivatives"

$$
\text { “ } \frac{\delta}{\delta \phi(z)} \int d w \phi^{n}(w)=n \phi^{n-1}(z) .
$$

Disregarding these "difficulties," we note that in (50) if $F$ is a polynomial in $\phi$ 's, then so is the expression in parentheses on the right side of (50). Use of this "identity" either lowers the degree of the polynomial, or introduces a power of $\lambda$. Thus iterated use of (50) generates each term in (48) in a finite number of steps. (This requires a moment's reflection.) We consider an artificial "example" in which

$$
\text { “ } S_{\mathrm{I}}^{\Omega}=\lambda \int \phi^{4}(x) d x "
$$

(we neglect renormalization terms). We seek an expression for $C_{2}(x, y)$, derived by iterated use of (50). One finds

$$
\begin{gathered}
C_{2}(x, y)=I_{1}+I_{2}+I_{3} \\
I_{1}(x, y)=c_{1} \int d z \int d w C_{0}(x, z)\left(C_{0}(z, w)\right)^{3} C_{0}(w, y) \\
I_{2}(x, y)=c_{2} \int d z \int d w C_{0}(x, z) C_{0}(z, w) C_{0}(w, y) C_{0}(w, w) C_{0}(z, z), \\
I_{3}(x, y)=c_{3} \int d z \int d w C_{0}(x, z) C_{0}(z, y)\left(C_{0}(z, w)\right)^{2} C_{0}(w, w)
\end{gathered}
$$


Most of these expressions will certainly be undefined (infinity)-we have neglected renormalization terms in $S_{I}^{\Omega}$ that "cancel" some of these infinities. The $c_{i}$ are numerical factors we choose not to keep track of. These three $I_{i}$, each a "Feynman integral," are associated to the "Feynman diagrams" in Figure 1(a), (b), and (c) respectively. Each propagator $C_{0}(\cdot, \cdot)$ is a line in the Feynman diagram. The coordinates are here indicated in the figures: $C_{0}\left(x_{1}, x_{2}\right)$ is a line from $x_{1}$ to $x_{2}$. Usually one does not indicate coordinates in the figures. We do not teach the reader the physicists' technique in "renormalizing" the divergent integrals; however, we point out (as the diligent reader may verify) that Wick ordering the $\phi^{4}$ in (53) formally cancels identically $I_{2}$ and $I_{3}$. $I_{1}$ is finite for two-dimensional theories, and infinite for three-dimensional theories. (The mass renormalization (44) is designed to handle this infinity.)

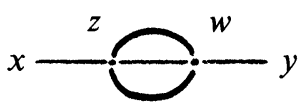

(a)

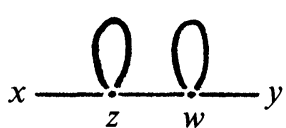

(b)

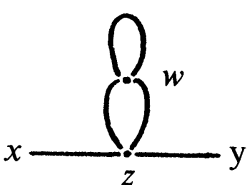

(c)

\section{FIGURE 1}

Physicists start with formal expressions such as in (55)-(57) and then apply their "renormalization prescriptions," rather than using limits in expressions such as (48). We have only touched the surface; much beautiful and difficult structure resides in individual perturbation series terms.

Suggested study. To someone who wished to work along the lines discussed herein, I would recommend they read in detail [1], work joint with G. Battle, that establishes the phase cell cluster expansion formalism. (One should replace the expansion functions described in $\S 2$ therein, by the o.n. basis $\left\{\psi_{k}^{\prime}\right\}$ of the present Appendix. This eliminates a small error in [1].) [7,8, and 9] contain some excellent expository material, particularly on cluster expansions, that should be digested before the details in [1]. I would recommend that at the same time they sit in on a one-year graduate course in quantum mechanics, followed by a one- or two-term course in quantum field theory, in a physics department. The same goal can be accomplished with greater difficulty by reading physics text books.

References [3,4, and 5] are pleasant reference books, and fun to browse in. In particular [3] of Glimm and Jaffe presents the more standard formalism of constructive quantum field theory. The reader of these notes will find in [3] the axioms of Euclidean field theory (due to Osterwalder and Schrader), and the recovery of the Minkowski field theory from the Euclidean field theory. These are also present in [4]. [3] also provides an introduction to the substantial literature in constructive quantum field theory, a guide to many more topics than we have discussed. At the technical level, we prefer the formalism of [1], to the more standard approach of [3]. 
Recent work of Brydges, Fröhlich, Sokal, and Spencer (see [2]) has exploited random walk techniques to study quantum field theories. This is an equally powerful, but more specialized, formalism than the present one. This is very beautiful work and should be studied. The important work of Feldman, Magnen, Rivasseau, and Sénéor is in a formalism similar to ours, and may be read in parallel.

We have offered enough suggestions to the reader, that with diligence he or she may enter the area of research. There will be much exciting but difficult work ahead as one attempts to tame the four-dimensional Yang-Mills theory. [The author has a sequence of preprints on the Yang-Mills Theory available.]

Appendix. The functions $u_{k}(x)$ are related to the functions $\psi_{k}^{\prime}(x)$ via (29) above. We herein describe the properties of the basis $\left\{\psi_{k}^{\prime}\right\}$, without giving their construction. We first describe a basis of functions $\left\{\psi_{k}\right\}$, the "ondelettes" of Y. Meyer [6], and then describe the $\psi_{k}^{\prime}$, a modification of the $\psi_{k}$ due to I. Daubechies.

Ondelettes. We consider compatible lattices $\mathscr{L}^{r}$ in $R^{d}$ of edge size $L_{r}=$ $1 / 2^{r}, r=0, \pm 1, \pm 2, \ldots$ We now state some of the properties of the o.n. basis $\left\{\psi_{k}\right\}$ developed by Y. Meyer associated to these lattices.

(1) Each $\psi_{k}$ is associated to some cube, in some $\mathscr{L}^{r}, r=r(k)$. There are the same number of $\psi_{k}$ associated to each cube. Given the set of $\psi_{k}$ associated to any cube, the set of $\psi_{k}$ associated to any other cube are the natural dilation, translation, and multiple of these $\psi_{k}$. More exactly, there are a number of functions $f_{1}, \ldots, f_{\alpha}$ such that the $\psi_{k}$ associated to cube $\gamma$ in $\mathscr{L}^{r}, r=r(\gamma)$, are

$$
L_{r(\gamma)}^{-d / 2} f_{i}\left(\frac{x-x^{(\gamma)}}{L_{r(\gamma)}}\right), \quad i=1, \ldots, \alpha,
$$

where $x^{(\gamma)}$ is the center of $\gamma$.

(2) $\psi_{k}$ is $C^{\infty}$, its Fourier transform is $C^{\infty}$ and of compact support.

(3) $\int \psi_{k} x^{\alpha}=0$, all $\alpha$, i.e. all the moments of the $\psi_{k}$ are zero.

$$
\left|\left(x-x^{(\gamma)}\right)^{\beta} D^{\alpha} \psi_{k}(x)\right| \leqslant c_{\alpha, \beta} L_{r}^{|\beta|-|\alpha|-d / 2}
$$

for compatible $r, \gamma, k$. That is, the $\psi_{k}$ fall off faster than any power, along with their derivatives, with distance from their associated cube (in the appropriate length scale).

Modified ondelettes. The basis of Y. Meyer above may be modified to yield a basis $\left\{\psi_{k}^{\prime}\right\}$ with the $\psi_{k}^{\prime}$ associated only to cubes in $\mathscr{L}^{r}, r \geqslant 0$. (We have a maximum cube size.) The modified basis satisfies the following properties:

(1) To a cube in $\mathscr{L}^{r}, r>0$, the associated functions are the same as above.

(2) To each cube in $\mathscr{L}^{0}$ the associated $\psi_{k}^{\prime}$ include the set of associated $\psi_{k}$, and a finite additional number of functions.

(3)' The additional functions associated to cubes in $\mathscr{L}^{0}$ need not have zero moments, but they satisfy (2) and (4) above. The additional functions associated to any cube in $\mathscr{L}^{0}$ are the natural translates of the additional functions associated to any other cube in $\mathscr{L}^{0}$. 


\section{REFERENCES}

1. G. Battle and P. Federbush, A phase cell cluster expansion for Euclidean field theories, Ann. Physics 142 (1982), 95-139.

2. D. Brydges, J. Fröhlich and A. Sokal, A new proof of the existence and nontriviality of the continuum $\phi_{2}^{4}$ and $\phi_{3}^{4}$ quantum field theories, Comm. Math. Phys. 91 (1983), 117.

3. J. Glimm and A. Jaffe, Quantum physics, Springer-Verlag, Berlin and New York, 1981.

4. B. Simon, The $p(\phi)_{2}$ Euclidean (quantum) field theory, Princeton Univ. Press, Princeton, N. J., 1974.

5. R. F. Streater and A. S. Wightman, PCT, spin and statistics, and all that, W. A. Benjamin, New York, 1964.

6. Y. Meyer, La transformation en ondelettes, preprint.

7. D. Brydges, What is a quantum field theory, Bull. Amer. Math. Soc. (N.S). 8 (1983), 31-40.

8. D. Brydges and P. Federbush, A new form of the Mayer expansion in classical statistical mechanics, J. Math. Phys. 19 (1978), 2064-2067.

9. D. Brydges, A short course on cluster expansions, Les Houches Summer School Notes (K. Osterwalder, ed.), 1984.

Department of Mathematics, University of Michigan, Ann Arbor, Michigan 48109 
